\title{
Fast Restoration of Colour Movie Scratches
}

\author{
Michal Haindl \& Jiří Filip \\ Institute of Information Theory and Automation \\ Academy of Sciences of the Czech Republic \\ 18208 Prague, Czech Republic
}

\begin{abstract}
This paper presents a new type of scratch removal algorithm based on a causal adaptive multidimensional multitemporal prediction. The predictor use available information from the neighbourhood of a missing multispectral pixels due to spectral, temporal and spatial correlation of video data but not any information from the failed pixels themselves.
\end{abstract}

\section{Introduction}

A huge amount of documentary or artistic movies is stored in different film archives throughout the world. Unfortunately every movie deteriorates with usage and time irrespective of any care it gets. Movies (on both optical and magnetic materials) suffer with blotches, dirt, sparkles and noise, scratches, missing or heavily corrupted frames, mold, flickering, jittering, image vibrations and some other problems [12]. As a result of ever growing demand for archive movies there is the urgent necessity for movie restoration from film and entertainment industry. Surprisingly the number of published algorithms in the area of movie restoration is rather small probably because of computational complexity of most restoration approaches. For each kind of the defect usually different kind of restoration algorithm is needed. Our attention in this paper is restricted to the scratch restoration problem. The scratch notion in this paper means every coherent region with missing data (simultaneously in all spectral bands) in a colour movie frame.

The problem how to recover lost or damaged image data is old - since the dawn of photography to the digital images or movies obtained directly using CCD cameras, radiotelescopes [8], scanners on board of satellites, etc. The simplest methods neglect temporal correlation and reconstruct single movie frames separately. Such methods used to reconstruct image scratches are mostly very simple and the reconstruction quality is seldom satisfactory. In this paper we called these methods "classical" and they are used for comparison. The simplest methods replace missing pixels by the local mean values (A), the median (B) or the motion compensated median [11]. Another method (C) linearly interpolates missing data using neighbours from both sides of unknown data section. These methods produce visible distortions on colour images. Even interpolation with higher order curves, such as quadratic fit, is of no help [2] - method (D). More sophisticated template-like methods suggested in [2] cannot be used for reconstruction of multi-spectral pixels with all spectral components missing what is the case of scratch reconstruction. Model-based methods use most often Markov random field type of models either in the form of wide sense Markov (regressive models) or strong Markov models (MRF). The noncausal regressive model used in [3],[4] has the main problem in time consuming iterative solution based on the conjugate gradient method. Similarly MRF based restoration methods [5], [9] require time consuming application of Markov chain Monte Carlo methods (MCMC). Besides this both approaches have to solve the problem when to stop these iterative processes. We have proposed a $2 \mathrm{D}$ regression model based reconstruction method [6], [7], which clearly outperforms these image reconstruction methods. The method was further improved in [8] to select a locally optimal predictor from two mutually competing symmetrical adaptive predictors for each pixel to be reconstructed. Similar 2.5D AR model was later used in [10], hower its degradation model requires time consuming Markov chain Monte Carlo iterations and is restricted to monospectral movies only. Another method based on the vector median model [1] enables colour scratch reconstruction but has similar disadvantage in the MCMC solution.

An optimal reconstruction resulting in visual disappearance of the scratch is difficult to achieve, however a much more precise replacement beyond the scope the current methods is needed. From this originated an idea to reconstruct missing data from available information due to large correlation between single image elements in the 4D spatiotemporal data space. In this paper we propose a fast multispectral movie reconstruction method based on a causal 3.5D AR data model. The method reconstructs missing 
multispectral (e.g., colour) pixels from available data in neighbouring frames and pixels from the corrupted frame as well. The algorithm requires information about objects motion between different movie frames, which is extensive research area itself beyond the scope of presented work. For our experiments we used the diamond search algorithm for fast block-matching motion estimation [13].
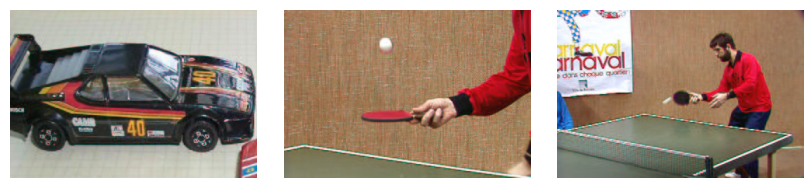

Figure 1. Experimental frames from the cars (left) and tennis sequences.

\section{The 3.5D Image Model}

Suppose $Y$ represents a digitized colour movie defined on a finite rectangular four dimensional $N \times M \times d \times \tau$ underlying lattice $I$, where $N \times M$ is the frame size, $d$ is the number of spectral bands (i.e., $d=3$ for usual colour movies) and $\tau$ is the overall number of frames in the film to be reconstructed. Corresponding pixel multiindex $r=\left\{r_{1}, r_{2}, r_{3}, r_{4}\right\}$ has the row, columns, spectral and time indices, respectively. All image data are assumed to be known except a set $(\mathcal{S})$ of unobservable multispectral pixels from some frame belonging to a scratch. The missing scratch data reconstruction from the topologically nearest known data in the lattice $I$ using temporal and spatial correlation in the neighbourhood generally requires a 4D model. Unfortunately parameters of such a AR model cannot be estimated analytically. However if we neglect mutual temporal correlations, i.e.,

$$
E\left\{e_{r_{1}, r_{2}, r_{3}, \bullet} e_{s_{1}, s_{2}, s_{3}, \bullet}^{T}\right\}=\operatorname{diag}\left[\sigma_{r_{3}}^{2}, \ldots, \sigma_{r_{3}}^{2}\right]
$$

$\forall s: s_{1}=r_{1}, s_{2}=r_{2}, s_{3}=r_{3}$ but nevertheless use different temporal multispectral pixels in a 3D AR model we obtain the 3.5D AR model which can be solved analytically under some additional acceptable assumptions. The notation - has the meaning of all possible values of the corresponding index. Suppose further that the multispectral multitemporal movie data can be represented by an adaptive causal 3.5 dimensional simultaneous autoregressive model:

$$
Y_{r_{1}, r_{2}, \bullet, r_{4}}=\gamma X_{r_{1}, r_{2}, \bullet, r_{4}}+e_{r_{\mathbf{1}}, r_{2}, \bullet, r_{4}} \quad \forall r \in I,
$$

where $Y_{r_{1}, r_{2}, \bullet, r_{4}}$ is a multispectral $d \times 1$ vector corresponding to a single multispectral pixel in the $r_{4}$-th frame, $X_{r_{1}, r_{2}, \bullet, r_{4}}=\left[Y_{r_{1}-s_{1}, r_{2}-s_{2}, \bullet, r_{4}-s_{4}}^{T}: \forall\left\{s_{1}, s_{2}, s_{4}\right\} \in I_{r_{1}, r_{2}, r_{4}}\right]^{T}$ either in the contextual neighbourhood $I_{r_{1}, r_{2}, r_{4}}$ or $/$ and in

$$
\gamma=\left[A_{1}, \ldots, A_{\eta}\right]
$$

is a $d \times d \eta$ parameter matrix, $I_{r_{1}, r_{2}, r_{4}} \subset I$ is a contextual neighbourhood and $\eta=\operatorname{card}\left\{I_{r_{1}, r_{2}, r_{4}}\right\}$. Data ordering in $X_{r_{1}, r_{2}, \bullet, r_{4}}$ corresponds to the arrangement of parameters in (2). The noise vectors $e_{r_{1}, r_{2}, \bullet, r_{4}}$ are assumed to be mutually uncorrelated zero mean white Gaussian, i.e.,

$$
E\left\{e_{r_{1}, r_{2}, \bullet, r_{4}} e_{s_{1}, s_{2}, \bullet, s_{4}}^{T}\right\}= \begin{cases}0 & \text { if } r \neq s \\ \Sigma & \text { otherwise }\end{cases}
$$

where $\Sigma$ is the noise covariance $d \times d$ matrix which is assumed to be constant but unknown to us.

The missing scratch data will be reconstructed from the topologically nearest known data in the lattice $I$ using temporal and spatial correlation in the neighbourhood. Scratch pixels are computed from the set of one-step-ahead predictions using the conditional mean predictor

$$
\tilde{Y}_{r}=E\left\{Y_{r_{1}, r_{2}, \bullet, r_{4}} \mid Y^{(r-1)}\right\}=\hat{\gamma}_{r-1} X_{r_{1}, r_{2}, \bullet, r_{4}}
$$

where $Y^{(r-1)}=\left\{Y_{r-1}, Y_{r-2}, \ldots, Y_{1}\right\}$ is the known process history and $\hat{\gamma}_{r-1}$ is the estimator of unknown model parameter matrix $\gamma(2)$. Simplified notation $r, r-1, \ldots$ in (3) and the process history denotes the multispectral process position in $I$ for fixed $r_{4}$, i.e., $r=\left\{r_{1}, r_{2}, \bullet, r_{4}\right\}, r-1$ is the location immediately preceding $r$, etc. A direction of movement on the underlying image sub-lattice corresponding to a corrupted frame is chosen in a way to erode the frame scratch.

If we assume the normal-Wishart (or alternatively Jeffreys) parameter prior then it was proven in [6] that the one-stepahead predictive posterior density to have the form of ddimensional Student's probability density with $\beta(r)-d \eta+$ 2 degrees of freedom, if $\beta(r)>d \eta$ then the conditional mean value is $(3)$, where

$$
\hat{\gamma}_{r-1}^{T}=V_{x x(r-1)}^{-1} V_{x y(r-1)} .
$$

The following notation is used in (4):

$$
\tilde{V}_{v w(r-1)}=\sum_{k=1}^{r-1} V_{k} W_{k}^{T}
$$

and $V_{v w(r-1)}=\tilde{V}_{v w(r-1)}+V_{v w(0)} \cdot V_{k}, W_{k}$ are either $X_{k}$ or $Y_{k}$ and $\beta(r)=\beta(0)+r-1=\beta(r-1)+1$, $\beta(0)>\eta-2$, and $\lambda_{(r)}=V_{y y(r)}-V_{x y(r)}^{T} V_{x x(r)}^{-1} V_{x y(r)}$ (see [7] for details). The model adaptation is introduced using the standard exponential forgetting factor technique in the parameter learning part of the algorithm [7]. Let us assume a set of AR models (1) $M_{1}, M_{2}, \ldots$ which can differ 
their exponential forgetting factor $\rho$. The optimal decision rule for minimizing the average probability of decision error chooses the maximum a posterior probability model, i.e., a model whose conditional probability given the past data is the highest one. The presented algorithm can be therefore completed [8] as:

$$
\begin{aligned}
\tilde{Y}_{r}^{i}=\tilde{\gamma}_{r-1}^{i, T} X_{i, r} \quad \text { if } \quad & p\left(M_{i} \mid Y^{(r-1)}\right)>p\left(M_{j} \mid Y^{(r-1)}\right) \\
& \forall j \neq i
\end{aligned}
$$

where $X_{i, r}$ are data vectors corresponding to $I_{r_{1}, r_{2}, r_{4}}^{i}$. Following the Bayesian framework, choosing uniform a priori model in the absence of contrary information, and assuming conditional pixel independence, the analytical solution has the form [8] $p\left(M_{j} \mid Y^{(r-1)}\right)=k \exp \left\{D_{j}\right\}$, where

$$
\begin{aligned}
D_{j}= & -\frac{d}{2} \ln \left|V_{x(r-1)}\right|-\frac{\beta(r)-d \eta+d+1}{2} \ln \left|\lambda_{(r-1)}\right| \\
& +\frac{d^{2} \eta}{2} \ln \pi \sum_{i=1}^{d}\left[\ln \Gamma\left(\frac{\beta(r)-d \eta+d+2-i}{2}\right)\right. \\
& \left.-\ln \Gamma\left(\frac{\beta(0)-d \eta+d+2-i}{2}\right)\right]
\end{aligned}
$$

and $k$ is a common constant. All statistics related to a model $M_{j} \quad \tilde{V}_{x y(r-1)}, \tilde{V}_{x x(r-1)},(10)$ are computed from data in $X_{j, r}$. The determinant $\left|V_{x x(r)}\right|$ as well as $\lambda_{r}$ can be evaluated recursively see [7]. If $A_{i}=$ $\operatorname{diag}\left[a_{1, i}, \ldots, a_{\nu, i}\right] \forall i$ then the $3.5 \mathrm{D}$ model reconstruction is identical with separately applied 2.5D model reconstruction on every monospectral scratch pixel component.

Let's assume a model movement towards the scratch. When the model reaches the scratch, the corrupted pixel prediction is evaluated. This is performed for each line in the scratch from top and bottom edge of the scratch using two symmetrical downwards and upwards moving models and their results are averaged. This helps to counterbalance artificial restriction on the contextual neighbourhood which has to be causal. Similarly another couple of models is moving in the opposite direction. Two computed predictions for each missing pixel have to be combined. Simple averaging is not appropriate, because each of both predictors has different distance from the last known original data and consequently it has also a different precision. Hence the exponential interleaving was used to weight the data influence from each side of the scratch as a function of the horizontal position of the predicted pixel on a scratch line to be reconstructed.

\section{Results and Conclusions}

The performance of the methods is compared on artificially created scratches, so that the original ("ideal") data are known. To prevent subjective visual comparison of the different methods results the mean absolute difference criterion between original and replaced pixel values is used.

$$
\overline{M A D}=\frac{1}{\nu d} \sum_{i=1}^{d} \frac{1}{\xi_{i}} \sum_{\forall r \in \mathcal{S}}\left|Y_{r_{1}, r_{2}, i, r_{4}}-\tilde{Y}_{r_{1}, r_{2}, i, r_{4}}\right|
$$

where $\nu=\operatorname{card}\{\mathcal{S}\}$ is the number of missing multispectral scratch pixels and $\xi_{i}$ ( $\xi_{i}=255 \forall i$ in our test movies $)$ is the number of quantization levels in the i-th spectral band. All evaluations are made on several frames (e.g., Fig.1)
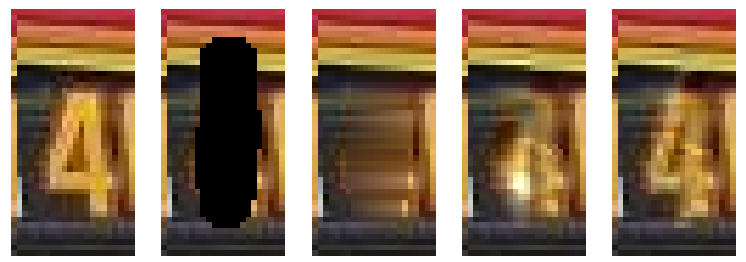

Figure 2. A car frame restoration (original, scratch, C method, 3D CAR, 3.5D CAR).

from two colour digital movies "cars" and "tennis". All selected frames are inside corresponding movie shots hence they have correlated neighbouring frames needed for the 3.5D presented data model. The model can be easily simplified into a $3 \mathrm{D}$ version (the multiindex has only three indices $\left.r=\left\{r_{1}, r_{2}, \bullet\right\}, I_{r_{1}, r_{2}}\right)$ and the scratch reconstruction is based on the reconstructed frame data only. Restoration results of 3D CAR and 3.5D CAR model are affected by the contextual neighbourhood $I_{r}$, by the exponential forgetting factor $\rho$, and by the length of the model history. The contextual neighbourhood has to be causal (in the reconstructed frame lattice $I$ subspace) to get analytical solution for the predictor and its cardinality influences computational complexity of the overall algorithm - the smaller cardinality $I_{r}$ has, the faster is the proposed method. Comparison results of the previously mentioned classical methods tested on the cars sequence frame are in Tab.1. The best results were obtained by the method $\mathrm{C}$ with contextual neighbourhood of two pixels on each side of a scratch. Both methods A and B suffer with clearly visible blurring tendency, while the methods $\mathrm{C}$ and $\mathrm{D}$ produce discernible columns in the reconstructed scratch. A significant improvement of the $3 \mathrm{D}$ model performance is obtained if the temporal information is included, i.e., using the 3.5D CAR model. Such information is natural to obtain from previous or / and following frame(s) for which we know all necessary data, due to high between-frame temporal correlation. Unfortunately it is not possible to simply pick up other frames data from the location corresponding to a reconstructed pixel in the 3.5D model, because of the motion present in the image sequence. So a motion estimation is needed between suc- 
Table 1. Results ( $\overline{M A D}$ criterion) of basic image restoration method on the cars sequence frame corrupted by scratch on Fig.2

\begin{tabular}{|l|c|c|c|c|c|c|}
\hline \multirow{2}{*}{ spectral } & \multicolumn{7}{|c|}{ method } \\
\cline { 2 - 7 } band & $\mathrm{A}$ & $\mathrm{B}$ & $\mathrm{C}$ & $\mathrm{D}$ & $3 \mathrm{D}$ & $3.5 \mathrm{D}$ \\
\hline Red & 0.228 & 0.250 & 0.208 & 0.239 & 0.149 & 0.092 \\
Green & 0.202 & 0.218 & 0.168 & 0.210 & 0.138 & 0.087 \\
Blue & 0.119 & 0.120 & 0.092 & 0.124 & 0.115 & 0.071 \\
\hline average & 0.183 & 0.196 & 0.156 & 0.191 & 0.134 & $\mathbf{0 . 0 8 3}$ \\
\hline
\end{tabular}

cessive frames if a speed of the object on the reconstructed scene is faster than 2-3 pixels between neighbouring frames.
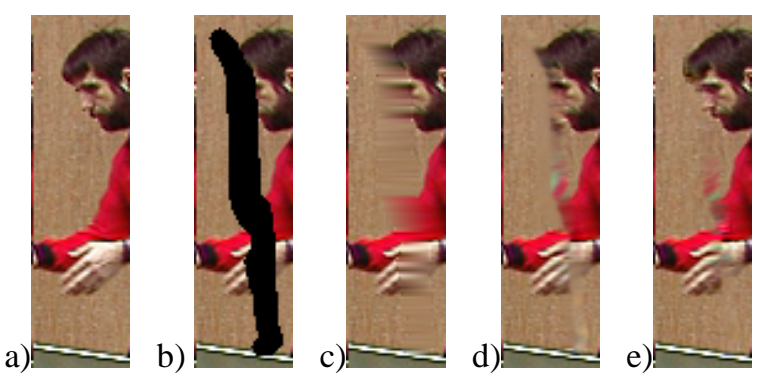

Figure 3. Scratch Fig.3-b) restoration in the 78th "tennis" (original (a), scratch (b), lin. regression (c), 3D CAR model (d), 3.5D CAR model (e)).

The motion estimation was performed by the method [13], however this algorithm demands more computation and prolongs the total time for restoration approximately five times in comparison with a 3D model. The motion estimation method results were sometimes unsatisfactory so for fully automatic restoration a better method is clearly required.

Because the exponential forgetting factor $\rho$ directly influences the process memory (and the speed of adaptation). The best values for tested scratches are $\rho=0.980-0.995$. The Fig. 3 example demonstrates significant reconstruction improvement over the classical methods (Tab. 1) and similar results were obtained also for the other experimental image frames. The test results of our algorithm are encouraging, the presented model was always superior over the classical methods not only using the $\overline{M A D}$ criterion but also visually. For sequences with fast moving objects, the method requires a fast and accurate motion detection which is only partially solved problem itself. However even if the reliable motion estimation is missing the method can be easily restricted to single frame support data with slightly compromised restoration quality. Finally if the method is used for isolated image pixels (or short lines perpendicular to the model movement) reconstruction then the predictor is computed from completely known data (without any ap- proximation) and the regression method performs much better than any of the previously published classical methods. The proposed method is fully adaptive, numerically robust and still with moderate computation complexity so it can be used in an on-line image acquisition / restoration system.

\section{Acknowledgements}

This research was supported by the Grant Agency of the Czech Republic under Grants 106/00/1715 and 102/00/0030.

\section{References}

[1] S. Armstrong, A. Kokaram, and P. Rayner. Reconstructing missing regions in colour images using multichannel median models. In European Conference on Signal Processing (EUSIPCO '98), volume 2, pages 1029-1032, September 1998.

[2] R. Bernstein, J. Lotspiech, H. Myers, H. Kolsky, and R. Lees. Analysis and processing of landsat-4 sensor data using advanced image processing techniques and technologies. IEEE Trans on Geosci., 22(3):192-221, 1984.

[3] R. Chellappa and R. Kashyap. Digital image restoration using spatial interaction models. IEEE Trans. Acoustics, Speeech and Sig. Proc., 30(3):284-295, June 1982.

[4] K. Deguchi and I. Morishita. Two-dimensional autoregressive model for the representation of random image fields. In Proc. ICPR Conf., pages 90-93, Munich, 1982. IEEE.

[5] D. Geman. Random fields and inverse problems in imaging. Springer, Berlin, 1990.

[6] M. Haindl and S. Šimberová. A Regression Type Image Destriping Method. In Biosignál '90, pages 74-75, Brno, June 1990. Dům techniky ČSVTS.

[7] M. Haindl and S. Šimberová. Theory \& Applications of Image Analysis, chapter A Multispectral Image Line Reconstruction Method, pages 306-315. World Scientific Publishing Co., Singapore, 1992.

[8] M. Haindl and S. Šimberová. A high - resolution radiospectrograph image reconstruction method. Astronomy and Astrophysics, Suppl.Ser., 115(1):189-193, January 1996.

[9] B. Jeffs and W. Pun. Simple shape parameter estimation from blurred observations for a generalized gaussian $\mathrm{mrf}$ image prior used in map restoration. In Proc. IEEE CVPR Conf., pages 465-468, San Francisco, 1996. IEEE.

[10] A. Kokaram. Motion Picture Restoration. PhD thesis, Cambridge University Engineering department, Trumpington Street, Cambridge CB2 1PZ, England, 1993.

[11] A. Kokaram. Motion Picture Restoration. Springer, London, Great Britain, 1998.

[12] P. Schallauer, A. Pinz, and W. Haas. Automatic restoration algorithm for $35 \mathrm{~mm}$ film. Journal of Computer Vision Research, 1(3):60-85, 1999.

[13] S. Zhu and K.-K. Ma. A new diamond search algorithm for fast block-matching motion estimation. IEEE Transactions on Image Processing, 9(2):287-290, February 2000. 